\title{
SOSTENIBILIDAD SOCIAL DE LOS SUBSISTEMAS PRODUCTIVOS DE TOMATE DE ÁRBOL (Solanum betaceum Cav) EN EL CANTÓN GUACHAPALA, PROVINCIA DE AZUAY - ECUADOR
}

\section{SOCIAL SUSTAINABILITY OF TOMATO TREE (Solanum betaceum Cav) PRODUCTION SUBSYSTEMS AT THE GUACHAPALA CANTON, PROVINCE OF AZUAY - ECUADOR}

\author{
Lourdes Díaz Granda ${ }^{1}$, Manuel Canto Sáenz ${ }^{2}$, Julio Alegre Orihuela ${ }^{3}$, Félix Camarena Mayta ${ }^{4}$ \\ y Alberto Julca Otiniano ${ }^{5}$
}

\begin{abstract}
Resumen
El tomate de árbol (Solanum betaceum Cav) es una planta nativa de América del Sur que está teniendo dificultades en mantenerse en las áreas de producción en Ecuador por problemas fitosanitarios, ocasionando la disminución de estas. Es una planta con bastante demanda en el cantón Guachapala, y últimamente se está expandiendo su producción. El objetivo de esta investigación fue evaluar la sostenibilidad social de las fincas productoras de tomate de árbol en el cantón Guachapala, Azuay-Ecuador. Se utilizó la “Metodología Multicriterio”. La población objetivo estuvo constituida por fincas con una plantación no menor de 12 meses de edad, contado a partir del trasplante a campo y en plena fructificación. Se trabajó con 52 productores y la información se obtuvo a través de encuestas estructuradas y adaptadas al medio. Se encontró que la producción del tomate de árbol en las fincas del cantón Guachapala es socialmente sustentable. Sin embargo, el acceso a la educación, el conocimiento y la conciencia ecológica, son variables que tienen valores críticos y podrían afectar, de manera negativa, su sostenibilidad en el futuro.
\end{abstract}

Palabras clave: farms, sustainability, indicators, Solanum betaceum.

\begin{abstract}
The tomato tree (Solanum betaceum Cav) is a native plant of South America. It is having difficulties to sustain the production areas of Ecuador due to phytosanitary problems that are causing the reduction of the production area. It is a plant with high demand at Guachapala Canton and recently, there is an increase of the production areas. The objective of this research was to evaluate the social sustainability of the tomato tree farms in the Guachapala Canton, Azuay-Ecuador. The "Multicriteria Methodology" was used. The target population consisted of farms with a plantation of no less than 12 months of age, counted from the transplant to the field and in full fructification. We worked with 52 producers and the information was obtained through structured and adapted surveys. It was found out that the production of tomato trees in the farms of Guachapala Canton, are socially sustainable. Nevertheless, the access to education and ecological awareness and awareness are variables that have critical values and could negatively affect sustainability in the future.
\end{abstract}

Key words: farms, sustainability, indicators, Solanum betaceum.

\section{Introducción.}

El tomate de árbol (Solanum betaceum Cav) es una planta nativa de América del Sur. Su centro de origen más probable son las selvas y los bosques de la zona ubicada en la reserva Tucumano-Boliviana al noroeste de Argentina y el sur de Bolivia. El norte de Perú y sur de Ecuador es considerado el centro de domesticación de la planta.

En Ecuador el tomate de árbol se cultiva en las provincias de Carchi, Imbabura, Pichincha, Cotopaxi, Tungurahua, Chimborazo, Bolívar, Cañar, Azuay y Loja (Revelo et al., 2004). En la provincia del Azuay los cantones de mayor producción de tomate de árbol son: Paute, Guachapala, El Pan y Sevilla de Oro. Hasta el año 2005, la superficie cultivada de tomate de árbol en el Azuay fue de 1800 ha, disminuyendo significativamente para el 2007, año en el que se tuvo 591 ha. Según Maita (2011), esta disminución ocurrió principalmente por el ataque de la antracnosis (Colletotrichum acutatum Simmonds). Actualmente el cultivo está nuevamente en expansión, habiéndose reportando 2397 ha a nivel nacional (INEC, 2010). No se conocen datos sobre la superficie del cultivo en el cantón Guachapala.

En el Cantón Guachapala la actividad económica se basa fundamentalmente en la agricultura, y se cultiva 
maíz, fréjol, arvejas, tomate riñón y tomate de árbol (GAD Guachapala, 2014). Este último tiene una demanda importante en la provincia, pero se sabe de la necesidad de mejorar los sistemas productivos de la especie, razón por la cual, es necesario realizar estudios de sostenibilidad de dichos sistemas productivos. En Ecuador, en los últimos años, se han realizado estudios para conocer la sostenibilidad de diversos sistemas agrícolas como café (Coffea arabica L.) en Jipijapa (Santistevan, 2013), limón (Citrus aurantifolia C.) en Santa Elena (Santistevan, 2015), y cacao (Theobroma cacao L.) y bambú (Guadua angustifolia k.) en Portoviejo (Palomeque, 2016). No se tienen estudios del tomate de árbol en Ecuador, por lo que este sería el primer trabajo en este tema. Los resultados podrán ayudar a planificar la producción y mejorar los índices de desarrollo social, contribuyendo de esta manera al desarrollo sostenible de dicha localidad. El objetivo de este trabajo fue evaluar la sostenibilidad social de las fincas productoras de tomate de árbol en el cantón Guachapala, Provincia de Azuay - Ecuador).

\section{Materiales y métodos.}

El estudio se desarrolló en la Provincia del Azuay, ubicada en la zona sur del callejón interandino, la provincia cuenta con 15 cantones; el cantón Guachapala es el más pequeño de la provincia, posee una superficie de $41.07 \mathrm{~km}^{2}$. Se ubica en la latitud sur $2^{\circ} 46^{\prime}$ y longitud oeste $78^{\circ} 42^{\prime}$. Limita al norte con Rio Paute que lo separa de las parroquias Tomebamba y Dug Dug, al sur con el cantón Guachapala, al este con el cantón El Pan y Sevilla de Oro y al oeste con el Rio Paute y cantón Paute (Figura 1). Su altura varía desde los 2200 metros hasta los 3280 metros sobre el nivel del mar. El clima predominante es templado, caliente en la parte baja del río Paute, templado en la cabecera cantonal y frío en la parte alta sector de Andacocha porque recibe las influencia de la Región Oriental, según la clasificación de Köpen el clima es Templado oceánico verano suave $(\mathrm{Cfb})$, la temperatura media anual es de $17^{\circ} \mathrm{C}$ la precipitación media anual es de 104 $\mathrm{mm}$, los meses más secos son diciembre y enero (57 $\mathrm{mm}$ ) y los meses de mayor precipitación son abril y octubre con registro promedio de $119 \mathrm{~mm}$. La humedad relativa promedio es de $83 \%$. Los suelos son de textura arcilloso, de tipo Dystropepts, Uderts, Histic Hydrandepts y Tropudalfs (GAD Guachapala. 2014). Los sectores de estudio fueron: Guachapala, Guablid, Sacre y Don Julo.

Metodología.

Para la investigación se utilizó el Análisis Multicriterio (Sarandón, 2002; Sarandón \& Flores, 2009). La información se obtuvo usando encuestas estructuradas y entrevistas. La metodología para evaluar la sustentabilidad social desarrollada por Sarandón y Flores (2009) consiste en realizar una serie de pasos que conducen a la obtención de un conjunto de sub indicadores adecuados para evaluar los

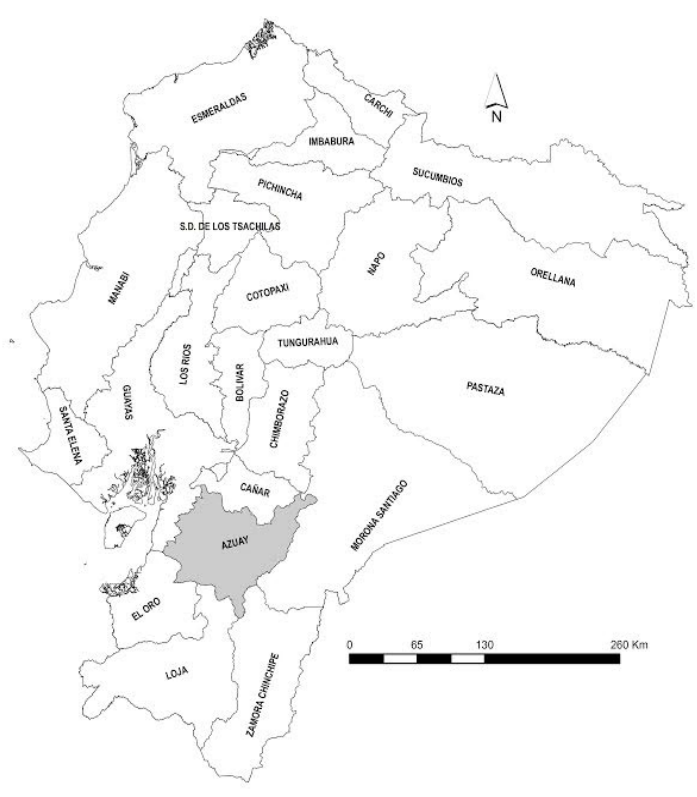

Figura 1. División política del Ecuador. Marcado en gris la provincia del Azuay, donde se encuentra el cantón Guachapala (elaborado por Tacuri. E. 2017. En base a la cartografía de SIGAGRO).

puntos críticos de la sustentabilidad de los agroecosistemas; es una metodología sencilla y de bajo costo que ayuda a obtener un indicador de fácil interpretación por cualquier observador (Sarandón, 2002).

Tamaño y selección de la muestra.

La población objetivo estuvo constituida por fincas productoras de tomate de árbol. Para ser considerada, deberían tener una plantación no menor de 12 meses de edad, contado a partir del trasplante a campo. Además, al momento de la investigación, deberían estar en plena fructificación. El total de productores tomateros, en los cuatro sectores estudiados, fueron 56; pero se usó una muestra $(\mathrm{n}=52)$, la misma que se calculó utilizando el simulador on line Fisterra (Pita Fernández, 2010). En la Tabla 1 se presenta la distribución de las fincas muestreadas en cada uno de los sectores estudiados.

Los datos obtenidos se analizaron usando los softwares SPSS y Excel. Se determinó el Índice de Sostenibilidad Social (ISS) por sector y por cantón, de acuerdo a la metodología de Sarandón et al. (2006), que se representó con gráfico de radar.

Tabla 1. Tamaño de la muestra por sector en el Cantón Guachapala.

\begin{tabular}{lcr}
\hline Sector & $\mathrm{N}^{\circ}$ de productores & Muestra \\
\hline Guachapala & 9 & 7 \\
Guablid & 13 & 13 \\
Don Julo & 22 & 20 \\
Sacre & 12 & 12 \\
TOTAL & 56 & 52 \\
\hline
\end{tabular}


Construcción de indicadores.

Para la construcción de indicadores se siguieron las recomendaciones de Sarandón et al. (2002). El indicador es la variable que puede ser medida con relación a un criterio específico (Lammerts van Bueren \& Blom, 1997). Los indicadores pueden ser cualitativos, cuantitativos (Astier et al., 2008). Un indicador de sostenibilidad lleva implícito un conjunto de valores y metas evocados en el concepto de sustentabilidad (Quiroga, 2001).

Indicadores seleccionados.

La selección de los sub indicadores, las variables a considerar en cada una de estas, y la valoración de las mismas, se realizó con la ayuda de nueve (09) expertos que trabajan en la zona, en el área productiva del cultivo; además de un (01) arquitecto y dos (02) productores de tomate. Este trabajo se complementó con las referencias bibliográficas, consultada para esta tarea. El detalle de este trabajo se describe a continuación:

A. Tenencia de la tierra.

La tenencia de la tierra es uno de los aspectos más importantes para que los agricultores tengan tranquilidad y puedan acceder a crédito para realizar su proceso productivo. Para construir este indicador se consideró el sub indicador Propiedad de la tierra.

$A_{1}$ - Propiedad de la tierra. Manera que asigna, dentro de las sociedades, los derechos de propiedad de la tierra; otorga el acceso a los derechos de utilizar, controlar y transferir la tierra, así como las pertinentes responsabilidades y limitaciones (FAO, 2003). La escala de valoración utilizada para este indicador fue: (4) Posee título de propiedad, (3) Título de propiedad en trámite, (2) Posesión, (1) Arrendamiento, (0) Préstamo. Se tomó como referencia la escala utilizada por el INEC (2010) y se ajustó en base a la opinión de expertos.

B. Satisfacción de necesidades básicas.

Un sistema sustentable es aquél en el cual los agricultores tienen aseguradas sus necesidades básicas, Comprende vivienda, educación, salud, servicios (Sarandón et al., 2006). Para construir este indicador se consideraron cuatro sub indicadores:

B1- Material de construcción de la vivienda. Se consideró el tipo de materiales predominantes para la construcción de la vivienda en la zona. Se consideró el tiempo de duración de los materiales, así como la frecuencia con la que se realiza el mantenimiento de los mismos en la vivienda. La escala de valoración utilizada para este indicador fue: (4) Ladrillo y cemento, (3) Bloque y cemento, (2) Adobe, (1) Tapial, (0) Madera, y se construyó en base a la opinión de expertos.

B2- Acceso a educación. La educación pública será gratuita y el Estado la financiará de manera oportuna, regular y suficiente. La distribución de los recursos destinados a la educación se regirá por criterios de equidad social, poblacional y territorial, entre otros
(Artículo 348. Constitución de la República del Ecuador, 2008). La escala de valoración utilizada para este indicador fue: (4) Superior, (3) Tecnológico, (2) Secundaria, (1) Primaria, (0) Ninguno. Se tomó como referencia la escala utilizada por el Instituto de Estadísticas y Censos (INEC, 2010) y se ajustó en base a la opinión de expertos.

B3- Acceso a salud, el artículo 362 de la Constitución de la República del Ecuador (2008) dice que los servicios públicos estatales de salud serán universales y gratuitos en todos los niveles de atención y comprenderán los procedimientos de diagnóstico, tratamiento, medicamentos y rehabilitación necesarios. La escala de valoración utilizada para este sub indicador fue construida en base a la distancia a la que se encuentra ubicado el dispensario médico más cercano: (4) < $1 \mathrm{~km}$, (3) 1.1 - $3 \mathrm{~km}$, (2) 3.1 - $5 \mathrm{~km}$, (1) $5.1-7 \mathrm{~km},(0)>7.1 \mathrm{~km}$. Se tomó como referencia la escala construida por Márquez \& Julca (2015) y se ajustó en base a la opinión de expertos.

B4- Acceso a servicios básicos, agua potable es el agua utilizada para los fines domésticos y la higiene personal, así como para beber y cocinar. Acceso al agua potable si la fuente de la misma se encuentra a menos de 1 kilómetro de distancia del lugar de utilización y si uno puede obtener de manera fiable al menos 20 litros diarios para cada miembro de la familia (OMS, 2016). La escala de valoración utilizada para este indicador fue: (4) Agua, luz y teléfono, (3) Agua y luz, (2) Agua y teléfono, (1) Luz y teléfono, (0) Teléfono. Se tomó como referencia la escala construida por Márquez \& Julca (2015) y se ajustó en base a la opinión de expertos.

C. Importancia de la conservación del ambiente.

La conservación del ambiente depende del conocimiento y conciencia ecológica y es fundamental para tomar decisiones adecuadas respecto a la conservación de los recursos (Sarandón et al., 2006), Para construir este indicador se consideraron dos sub indicadores:

C1- Conocimiento y conciencia ecológica. La escala de valoración utilizada para este indicador fue: (4) Muy alto conocimiento y conciencia ecológica, conoce sus fundamento, (3) Alto conocimiento y conciencia ecológica, aplica diariamente en su parcela, (2) Mediano conocimiento y conciencia ecológica, visión parcial, (1) Poco conocimiento y conciencia ecológica, desconoce las consecuencias de algunas prácticas, (0) No tiene conocimiento ni conciencia ecológica, realiza prácticas agresivas al medio. Se tomó como referencia la escala construida por Márquez \& Julca (2015) y se ajustó en base a la opinión de expertos.

C2- Grado de instrucción del propietario. Grado más elevado de estudios realizados o en curso, sin tener en cuenta si se han terminado o están incompletos (EUSTAT, 2011). La escala de valoración utilizada para este indicador fue: (4) Superior, (3) Tecnológico, 
(2) Secundaria, (1) Primaria, (0) Ninguna. Se tomó como referencia la escala utilizada por el INEC (2010) y se ajustó en base a la opinión de expertos.

Estandarización y ponderación de los sub indicadores.

La estandarización de sub indicadores se realizó con el fin de comparar las fincas y facilitar el análisis de la sostenibilidad social, los datos fueron estandarizados, mediante la transformación a una escala, para cada indicador, de 0 a 4 , siendo 4 el valor de mayor sostenibilidad y 0 el más bajo. Todos los valores, independientemente de su valor original, se transformaron o adecuaron a esta escala. Esto posibilitó la integración de varios indicadores de distinta naturaleza en otros más sintéticos o robustos. Posteriormente, los indicadores fueron ponderados multiplicando el valor de la escala por un coeficiente de acuerdo a la importancia relativa de cada variable respecto a la sostenibilidad. Este coeficiente multiplica, tanto el valor de las variables que forman el indicador, como el de los indicadores para construir indicadores de mayor nivel o índices. La ponderación es un paso que se hace por consenso con los expertos y agricultores como lo recomiendan Roming et al. (1996) y Letroy et al. (2000).

Las bases de datos construidas con los datos obtenidos en campo se manipularon con Microsoft Excel 2010 y SPSS versión 24. El indicador que mide el grado de cumplimiento de la sostenibilidad de la dimensión social (ISS), obtiene como valor máximo 4, se valora como sustentable cuando alcanza un valor igual o mayor al valor medio de la escala, es decir, 2 (Sarandón et al., 2006), lo que se puede observar en los diagramas de radar.

Para el cálculo del Indicador de Sostenibilidad Social (ISS), los sub indicadores recibieron diferentes pesos, los sub indicadores de mayor peso (dos) fueron: Acceso a educación (B2), Acceso a salud (B3) y Acceso a servicios básicos (B4). La fórmula aplicada se divide entre tres porque se estudiaron tres indicadores, así:

$$
I S S=\frac{A 1+\frac{B 1+2 B 2+2 B 3+2 B 4}{7}+\frac{C 1+C 2}{2}}{3}
$$

\section{Resultados y discusión.}

En la Tabla 2 se muestra que el sistema de producción de tomate de árbol es sostenible socialmente puesto que todos los sectores superan el valor de 2, al igual que la sostenibilidad por cantón (2.53). Por lo tanto, las fincas productoras de tomate de árbol con socialmente sostenibles de acuerdo a la metodología de Sarandón et al. (2006).

Sin embargo, algunas variables presentan valores bajos que se deben analizar porque podrían afectar la sostenibilidad en el futuro. Así, tenemos el caso del Grado de Instrucción del Propietario que posee el valor más bajo (1.20) y es inferior al valor promedio de 2. Lo mismo ocurre con el Conocimiento y Conciencia Ecológica (1.33), que también tiene un valor menor al promedio de 2. Resultados similares han sido reportados en otras zonas rurales, como en el valle de Cañete en Perú (Collantes, 2016), con fincas productoras de palto y mandarina; también en fincas productoras de café en Jipijapa, Ecuador (Santistevan, 2013), aunque también han sido reportados valores mayores para esta variable en fincas productoras de cacao en Ecuador (Palomeque, 2016).

El Acceso a Educación (1.61) tiene valor bajo y difiere de lo reportado por Palomeque (2016) para fincas productoras de cacao en Manabí, Ecuador; y Collantes (2016), para fincas productoras de palto y mandarina en Cañete, Perú. El Acceso a Salud (2.45) tiene un valor superior a 2, lo que difiere de los datos encontrados por Santistevan (2013), que reporta valor de cero, y tampoco coincide con los resultados encontrados por Palomeque (2016) y Collantes (2016), quienes obtuvieron un valor de 4. El tipo de Material de Construcción de la Vivienda (2.59) tiene un valor superior al encontrado por Santistevan (2013), quien indica tener valor de 2, a diferencia de Palomeque (2016), quien manifiesta tener valor de 4. El Acceso a Servicios Básicos (3.50) indica que la mayoría de viviendas cuentan con servicios de luz, agua y teléfono, valor superior al reportado por Santistevan (2013), quien indica tener valor de 2 , y cercano al encontrado por Palomeque (2016), que obtuvo valor de 4 . El valor obtenido para Propiedad de la Tierra (3.79) evidencia que la mayoría de propietarios tiene legalizado el título de propiedad del predio. La Figura 2 muestra, de

Tabla 2. Resumen de la evaluación de la sostenibilidad social de las fincas de

\begin{tabular}{|c|c|c|c|c|c|c|c|c|}
\hline \multirow[b]{2}{*}{ Sector } & \multicolumn{7}{|c|}{ Sub indicadores Socio Culturales } & \multirow{2}{*}{$\begin{array}{c}\text { Indicador de } \\
\text { Sostenibilidad } \\
\text { Social (ISS) }\end{array}$} \\
\hline & $\begin{array}{c}\text { PT } \\
\text { (A1) }\end{array}$ & $\begin{array}{c}\mathrm{MCV} \\
\text { (B1) }\end{array}$ & $\begin{array}{c}\mathrm{AE} \\
(\mathrm{B} 2)\end{array}$ & $\begin{array}{c}\text { AS } \\
\text { (B3) }\end{array}$ & $\begin{array}{l}\text { ASB } \\
\text { (B4) }\end{array}$ & $\begin{array}{l}\mathrm{CCE} \\
(\mathrm{C} 1) \\
\end{array}$ & $\begin{array}{l}\text { GIP } \\
(\mathrm{C} 2)\end{array}$ & \\
\hline Guachapala & 3.14 & 2.14 & 2.00 & 2.14 & 3.57 & 1.57 & 1.29 & 2.36 \\
\hline Guablid & 4.00 & 2.85 & 2.00 & 1.92 & 4.00 & 1.23 & 1.23 & 2.63 \\
\hline Sacre & 4.00 & 2.58 & 1.17 & 2.75 & 3.67 & 1.23 & 1.08 & 2.56 \\
\hline Don julo & 4.00 & 2.80 & 1.25 & 3.00 & 2.75 & 1.30 & 1.20 & 2.55 \\
\hline Promedio & 3.79 & 2.59 & 1.61 & 2.45 & 3.50 & 1.33 & 1.20 & 2.53 \\
\hline
\end{tabular}
productores de tomate de árbol en el Cantón Guachapala. Ecuador.

Leyenda: $\mathrm{PT}=$ Propiedad de la tierra, $\mathrm{MCV}=$ Material de construcción de la vivienda, $\mathrm{AE}=$ Acceso a educación, $\mathrm{AS}=$ Acceso a salud, ASB= Acceso a servicios básicos, $\mathrm{CCE}=$ Cocimiento y conciencia ecológica, $\mathrm{GIP}=$ Grado de instrucción del propietario. 


\section{Guachapala}

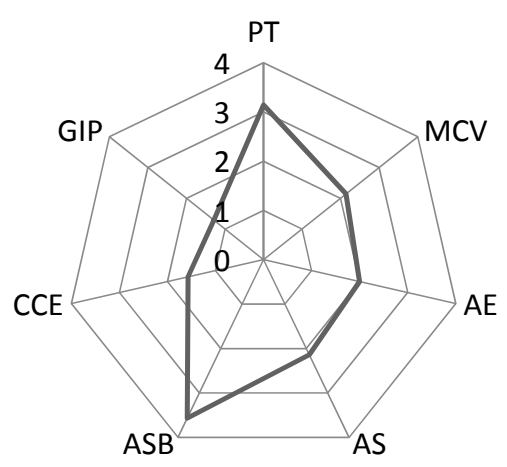

Sacre

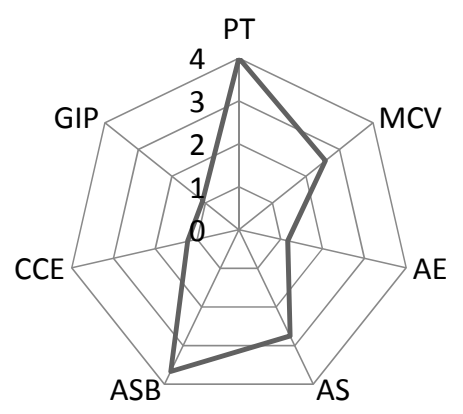

Guablid

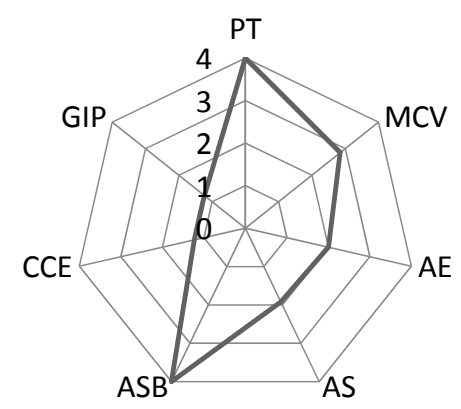

Don Julo

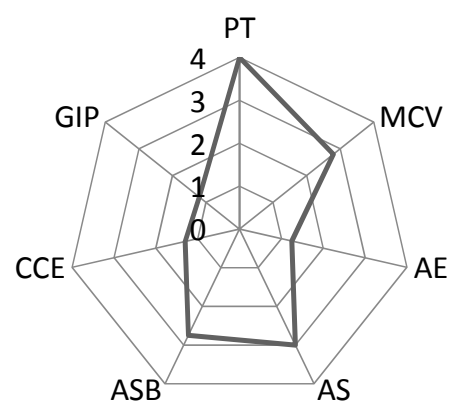

Figura 2. Representación gráfica de la Sostenibilidad Social en: Guachapala, Guablid, Sacre y Don Julo (PT = Propiedad de la tierra, $\mathrm{MCV}=$ Material de construcción de la vivienda, $\mathrm{AE}=$ Acceso a educación, $\mathrm{AS}=$ Acceso a salud, $\mathrm{ASB}=$ Acceso a servicios básicos, $\mathrm{CCE}=$ Cocimiento y conciencia ecológica, GIP= Grado de instrucción del propietario).

manera gráfica, los puntos críticos de la sostenibilidad social en cada uno de las localidades investigadas. Se observa claramente que el Grado de instrucción del propietario, conocimiento y conciencia ecológica, son puntos críticos que, como ya se ha dicho, podrían afectar la sostenibilidad en el futuro. Si bien el ISS para cada una de estas localidades es mayor a 2, se observa que la sostenibilidad alcanzada por Guachapala, Guablid y Sacre se explicaría, principalmente, por los mismos factores (Propiedad de la tierra y Acceso a servicios básicos); y mayormente, la Propiedad de la tierra, explicaría los resultados en Don Julo. Santistevan (2015) señala que el concepto de sustentabilidad es complejo y, como consecuencia, los "puntos críticos" para alcanzar la sustentabilidad pueden cambiar de un sistema de producción a otro; es lógico encontrar algunas diferencias entre las fincas evaluadas. En este caso, y con mayor razón, las diferencias también se muestran entre localidades.

\section{Conclusiones.}

Las fincas productoras de tomate de árbol, en el cantón Guachapala, son socialmente sustentables. Pero el acceso a la educación y el conocimiento, y conciencia ecológica, son variables que tienen valores críticos y podrían afectar, de manera negativa, la sostenibilidad en el futuro.

\section{Agradecimientos.}

A todos los agricultores participantes en la investigación por su paciencia y colaboración para llevar a cabo este estudio, y por su constancia en el sacrificado proceso productivo de cultivar alimentos para el pueblo ecuatoriano.

A los profesionales del GAD Guachapala, INIAP y Universidad de Cuenca, por sus aportes técnicos y científicos. 


\section{Literatura citada.}

Astier M., Masera O. R. \& Galván-Miyoshi Y. 2008. Evaluación de la sustentabilidad. Un enfoque dinámico y multidimensional. Formulación de indicadores sustentables para evaluación de sustentabilidad de sistemas de manejo complejos. Ediciones Mundiprensa. Capítulo 5: 73-92.

Collantes R., 2016. Sustentabilidad de los agroecosistemas de palto (Persea americana Mill.) y mandarina (Citrus spp.) en el valle de Cañete, Lima, Perú. Tesis Doctorado en Agricultura Sustentable. Escuela de Post Grado de la Universidad Nacional Agraria La Molina. Lima-Perú. 58 pp.

Constitución de la República del Ecuador. 2008. p 9.

EUSTAT. 2011. Censo de población y vivienda.

FAO. 2003. Tenencia de la tierra y desarrollo rural. ISBN 925-304846-8.

GAD Guachapala. 2014. Plan de desarrollo y ordenamiento territorial "Cantón Guachapala” Gobierno Autónomo Descentralizado Municipal de Guachapala 2014-2027.

INEC (Instituto Nacional de Estadísticas y Censos). 2010. Resultados del Censo de Población y Vivienda. Fascículo provincial de Azuay. Quito, Ecuador. 7 pp.

Lammerts Van Bueren E. \& Blom E. 1997. Hierarchical framework for the formulation for sustainable forest management standards: Principles, criteria and indicators. Tropenbos Foundation, Wageningen (The Netherlands). $82 \mathrm{pp}$.

Letroy R. D. B., Bechstedt H. D. \& Rais M. 2000. Indicators for sustainable land management based on farmer survey in Vietnam, Indonesia and Thailand. Agriculture, Ecosystem and Enrironment, 81(1):137-146.

Maita S. 2011. Manejo del "ojo de pollo" o antracnosis (Colletotrrichum acutatum Simmonds) en el cultivo de tomate de árbol (Solanum betaceum Cav). Cuenca, Ecuador. p 3-5.

Márquez F. \& Julca A. 2015. Indicadores para evaluar la sustentabilidad en fincas cafetaleras en Quillabamba. Revista de la Facultad de Ingeniería USIL Saber y hacer 2(1):128-137.

OMS (Organización Mundial de la Salud). 2016. Agua, Saneamiento y Salud (ASS). Agua potable salubre y saneamiento básico en pro de la salud. 14 pp.

Palomeque M. 2016. Sustentabilidad en sistemas agrícolas de limón (Citrus aurantifolia C.), cacao (Theobroma cacao
L.) y bambú (Guadua angustifolia k.), en PortoviejoEcuador. Tesis Doctorado en Agricultura Sustentable. Escuela de Post Grado de la Universidad Nacional Agraria La Molina. Lima-Perú. 79 pp.

Pita Fernández S. 2010. Determinación del tamaño muestral. Unidad de Epidemiología Clínica y Bioestadística. Complexo Hospitalario Universitario de A Coruña CAD ATEN PRIMARIA 1996; 3: 138-14.

Quiroga R. 2001. Indicadores de sostenibilidad y desarrollo sostenible: estado del arte y perspectivas. Serie Manuales 16 CEPAL, Naciones Unidas. (Manual producido por el Proyecto Evaluación de la Sostenibilidad den América Latina y El Caribe, PESALC). Santiago, Chile. 116 pp.

Revelo J., Pérez E. \& Malla, M. 2004. Cultivo ecológico del tomate de árbol en Ecuador. Texto de consulta del estudiante. Proyecto: IQ-CVÑN097. Quito - Ecuador. 87 pp.

Roming D. E., Garlynd M. J. \& Jarris R. F. 1996. Farmerbased assessment of soil quality: a soil health scorecard. Pp.39-60. in J.W. Doran and A.J. Jones (Eds.). Methods for Assessing soil quality: SSSA Special Publication $\mathrm{N}^{\circ}$. 49 SSSA, Madison. 30 pp.

Santistevan M. 2013. Sustentabilidad de Fincas Cafetaleras en Jipijapa Manabí, Ecuador. Tesis Maestría en Agricultura Sustentable. Universidad Nacional Agraria La Molina. Lima. Perú. 117 pp.

Santistevan M. 2015. Sustentabilidad del cultivo de limón en San Elena, Ecuador. Tesis Doctorado en Agricultura Sustentable. Universidad Nacional Agraria La Molina. Lima. Perú. 73 pp.

Sarandón S. 2002. El desarrollo y uso de indicadores para evaluar la sustentabilidad de los agroecosistemas. En Agroecología. El camino hacia una agricultura sustentable. Ediciones Científicas Americanas, Capitulo 20: 393-414.

Sarandón S. \& Flores C. 2009. Evaluación de la sustentabilidad en Agroecosistemas: Una propuesta metodológica. Agroecología 4: 19-28.

Sarandón S. J., Zuluaga M. S., Cieza R., Gómez C., Janjetic L. \& Negrete E. 2006. Evaluación de la sustentabilidad de sistemas agrícolas de fincas en Misiones, Argentina, mediante el uso de indicadores. Revista Agroecología 1:19-28.

\footnotetext{
${ }^{1}$ Universidad de Cuenca. Facultad de Ciencias Agropecuarias. Carrera de Ingeniería Agronómica. Programa Doctorado Agricultura Sustentable. e-mail: lourdes.diaz@ucuenca.edu.ec.

${ }^{2}$ Universidad Nacional Agraria La Molina. Programa Doctorado Agricultura Sustentable. Facultad de Agronomía. e-mail: mcanto@lamolina.edu.pe.

${ }^{3}$ Universidad Nacional Agraria La Molina. Programa Doctorado Agricultura Sustentable. Facultad de Agronomía. e-mail: jalegre@lamolina.edu.pe.

${ }^{4}$ Universidad Nacional Agraria La Molina. Programa Doctorado Agricultura Sustentable. Facultad de Agronomía. e-mail: camafe@lamolina.edu.pe.

${ }^{5}$ Universidad Nacional Agraria La Molina. Programa Doctorado Agricultura Sustentable. Facultad de Agronomía. e-mail: ajo@lamolina.edu.pe.
} 